\title{
Long-term clinical efficacy of ostial stenting to the left anterior descending artery with deep caudal projection
}

\author{
Kenji Sadamatsu*, Yuya Yoshidomi, Keiki Yoshida \\ Department of Cardiology, Saga-Ken Medical Centre Koseikan, Saga, Japan \\ Email: "k-sadamatsu@umin.ac.jp \\ Received 3 September 2013; revised 5 October 2013; accepted 15 October 2013 \\ Copyright (C) 2013 Kenji Sadamatsu et al. This is an open access article distributed under the Creative Commons Attribution License, \\ which permits unrestricted use, distribution, and reproduction in any medium, provided the original work is properly cited.
}

\begin{abstract}
Purpose: Overlapping and foreshortening on conventional coronary angiography is one reason why the presence of ostial lesions in the left anterior descending artery (LAD) is an independent predictor of insegment restenosis. Our previous study using 3-dimensional reconstruction coronary angiography demonstrated the superiority of a deeper caudal projection in clearly showing the ostium of the LAD compared to the standard right or left anterior oblique caudal view. Therefore, the aim of this study was to determine the short- and long-term efficacy of the ostial stenting technique using a deep caudal projection angle. Methods: A total of 30 consecutive patients who underwent stent deployment to treat LAD lesions positioned at the proximal edge of the stent in the ostium with a deep caudal projection were analyzed retrospectively. Results: The projection of right anterior oblique caudal $40^{\circ}$ was used in 26 patients and that of left anterior oblique caudal $40^{\circ}$ was used in four patients. Intravascular ultrasound examinations showed complete coverage of the ostium by the stent in 29 patients. In one patient, the stent did not cover the ostium. The mean protrusion of the stent over the ostium was $0.66 \pm 0.85 \mathrm{~mm}$. During the follow-up period (1203.5 [982 - 1329] days), the rate of target lesion failure was $16.7 \%$, and there were no cases with in-stent restenosis of the main branch in which a drug-eluting stent covering the ostium of the LAD was successfully deployed. Conclusions: Focal stent placement using a deep caudal projection is a feasible and effective therapeutic strategy for treating ostial lesions in the LAD.
\end{abstract}

Keywords: Coronary Artery Disease; Three-Dimensional Imaging; Angiography; Bifurcation Lesions; Intravascular Ultrasound

${ }^{*}$ Corresponding author.

\section{INTRODUCTION}

Performing percutaneous coronary intervention to the ostial left anterior descending artery (LAD) is challenging, even in the era of drug-eluting stents, because the presence of ostial lesions is an independent predictor of in-segment restenosis [1]. While such restenosis is associated with numerous factors, among which incomplete lesion coverage seems to be the most critical one, which thus highlights the technical difficulties encountered in achieving precise stent positioning in the ostium [2]. These technical difficulties primarily result from foreshortening of the LAD and overlapping of the LAD and the left circumflex artery (LCX) on conventional coronary angiography. Therefore, focal stenting, which is often attempted in patients with ostial lesions, may result in incomplete lesion coverage or protrusion of the proximal stent margin into the ostium of the circumflex artery [3]. Furthermore, the presence of plaque and/or carina shift with compromise of the adjacent vessels [4-6] and the difficulty of performing repeat intervention for in-stent restenosis are also important issues that lead to many patients with ostial lesions being referred for bypass surgery. On the other hand, favorable outcomes of unprotected left main coronary artery stenting with drugeluting stents have been recently reported, and the crossover stenting technique has emerged as a new stenting strategy for treating ostial LAD lesions. The easier stent positioning guaranteeing full coverage of the lesions achieved with this method may lead the cross-over stenting technique to become a mainstream therapy for treating ostial LAD lesions [7-10]. However, there are some disadvantages associated with the cross-over technique. Jailing the ostium of the LCX with the stent is the primary problem and requires the use of the kissing balloon inflation technique in almost all cases [8,9]. This complex inflation technique results in longer procedure and fluoroscopic times and the use of larger contrast volumes. In order to cover the area from the left main trunk to the 
$\mathrm{LAD}$, the stent length must become longer [10]. A discrepancy in lumen size between the left main trunk and the ostial LAD is a concern of incomplete stent apposition in the left main trunk. In spite of these disadvantages, the cross-over stenting technique is often adopted for fear of achieving incomplete lesion coverage with ostial stenting.

Recently, we investigated the optimization of the projection angle to clearly show the ostium of the LAD using 3-dimensional reconstruction coronary angiography and demonstrated the superiority of a deeper caudal projection over the standard right or left anterior oblique caudal view [11]. Therefore, the use of focal ostial stenting to the LAD with a deep caudal projection may be able to overcome concerns of incomplete lesion coverage. The aim of this study was to determine the shortand long-term efficacy of the ostial stenting technique to the LAD with a deep caudal angle.

\section{METHODS}

\subsection{Study Population}

The institutional review board approved this study. Written informed consent was obtained from all patients. All patients were at least 18 years of age and presented with either stable/unstable angina or documented silent myocardial ischemia. A total of 30 consecutive patients who underwent stent deployment to treat LAD lesions positioned at the proximal edge of the stent in the ostium with a deep caudal projection between April 2008 and June 2009 were analyzed retrospectively. In addition to those with significant LAD ostial lesions, patients with diffuse significant proximal LAD lesions originating from the ostium in whom the ostial lesions were not significant were also included. Patients with significant left main lesions and LCX ostial lesions were excluded. The final decision to perform ostial stenting was left to the operators.

\subsection{Techniques}

All procedures were performed using standard techniques. Unfractionated heparin at a dose of 10,000 units was administered at the start of the procedures. Dual antiplatelet therapy with aspirin at a dose of $100 \mathrm{mg} /$ day and clopidogrel at a dose of $75 \mathrm{mg} /$ day or ticlopidine at a dose of $200 \mathrm{mg} /$ day was recommended to be continued for at least 12 months in patients with drug-eluting stents and six months in patients with bare metal stents.

A deep caudal projection (right anterior oblique or left anterior oblique caudal $40^{\circ}$ ) was used to implant the stent positioned at the proximal edge of the stent in the LAD ostium. We previously demonstrated that the right anterior oblique caudal $40^{\circ}$ projection is optimal for stent placement in patients with ostial LAD lesions in most cases [11]. As a result, we first selected the right anterior oblique projection. If the projection did not work, we changed it to the left anterior oblique caudal $40^{\circ}$. In addition, we used intravascular ultrasound (IVUS) in all cases and confirmed that the position of the IVUS transducer showing the LAD ostium was just in the LAD ostium on angiography with a deep caudal projection. Under the guidance of the images, we positioned the stent to guarantee complete LAD ostium scaffolding with minimum protrusion of the stent at the level of the LCX ostium. A representative case is shown in Figure 1.

\subsection{Patient Follow-Up}

The patients were followed up at six months after the procedure and once a year routinely, unless symptoms or events required earlier consultation. Angiographic follow- up at eight months was encouraged for all patients. Follow-up data were collected until October 2012. Target lesion failure was defined as cardiac death, target vessel-related nonfatal myocardial infarction, target lesion revascularization or stent thrombosis.

\subsection{Statistical Analysis}

Quantitative data are presented as the mean value \pm SD or the median [interquartile range], and qualitative data are presented as frequencies. The Kaplan-Meier method and logrank test were used to assess the time to target lesion failure. All statistical analyses were performed with the SPSS software program (SPSS, Inc., Chicago, Ill).

\section{RESULTS}

The clinical and lesion characteristics of the patients are shown in Table 1. Stenosis of the ostial lesions was not severe because we included not only patients with significant ostial lesions, but also patients with diffuse LAD lesions requiring coverage with the stent to the ostium. The procedural characteristics are shown in Table 2. All procedures were successful. In most cases, the right anterior oblique caudal $40^{\circ}$ projection was used. Stent deployment following pre-dilation without post-dilation was the most frequent technique. Plaque or carina shift to the LCX ostium was minimal, and we dilated the LCX ostium with a conventional balloon in only one patient. Bare metal stents were used in two patients with acute myocardial infarctions. The IVUS findings (Table 3) demonstrated that the stents were deployed precisely in the LAD ostium with minimal protrusion to the left main trunk, except in one patient.

The median period of clinical follow-up was 1203.5 [982-1329] days. 1-, 2- and 3-year follow-up was completed in $29(96.7 \%), 28(93.3 \%)$ and 21 (70.0\%) patients, respectively. Follow-up angiography was performed in 


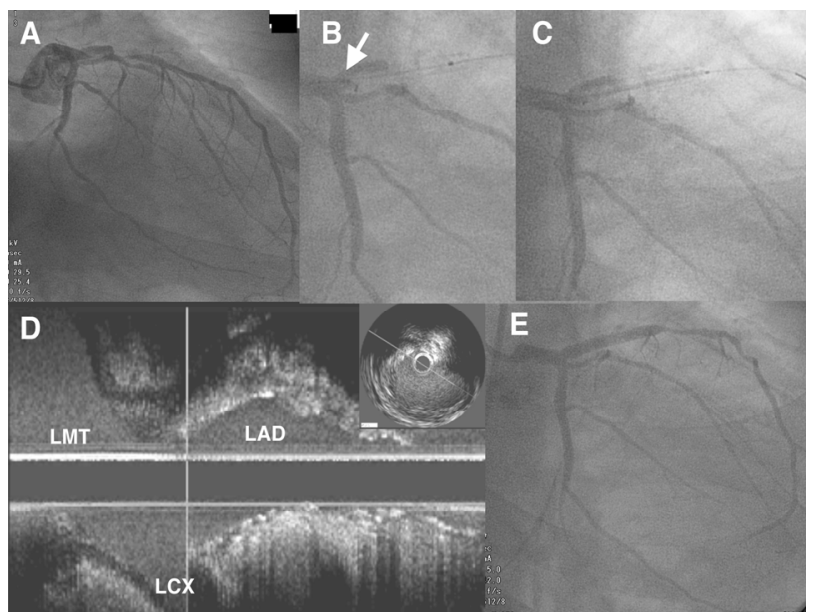

Figure 1. A representative case. A control angiogram (right anterior oblique $30^{\circ}$ - caudal $30^{\circ}$ ) showing overlap of the proximal left anterior descending artery and the circumflex artery (A). A deep caudal view (right anterior oblique $15^{\circ}$ caudal $40^{\circ}$ ) clearly demonstrating appropriate positioning of the intravascular ultrasound probe (arrow), which was placed in the ostium of the left anterior descending artery (B). A stent was deployed under the same projection (C). Intravascular ultrasound images revealing that the proximal edge of the stent was accurately deployed in the ostium (D). The lesion was successfully dilated without any complications (E).

Table 1. Clinical and lesion characteristics of patients.

\begin{tabular}{cc}
\hline Age (years) & $70.5 \pm 9.6$ \\
Male & $20(67)$ \\
Risk factors & \\
Diabetes mellitus & $13(43)$ \\
Hypertension & $25(83)$ \\
Dyslipidemia & $21(70)$ \\
Smoking & $9(30)$ \\
Diagnosis & $3(10)$ \\
Acute myocardial infarction & $5(17)$ \\
Unstable angina & $22(73)$ \\
Stable coronary disease & \\
Quantitative coronary angiography & $10.50 \pm 6.70$ \\
Baseline & $2.89 \pm 0.55$ \\
Lesion length (mm) & $1.50 \pm 0.63$ \\
LAD reference diameter (mm) & $47.0 \pm 22.2$ \\
Minimal lumen diameter (mm) & \\
Diameter stenosis (\%) & $3.01 \pm 0.53$ \\
Post-procedure & $2.60 \pm 0.72$ \\
Minimal lumen diameter (mm) & \\
Diameter at the LCX ostium (mm)
\end{tabular}

Data are expressed as mean \pm SD or number (percentage). LAD denotes left anterior descending artery; LCX, left circumflex artery.

$27(90.0 \%)$ patients, while myocardial ischemia was examined on myocardial scintigrams in the other three patients. During the follow-up period (Table 4), acute myocardial infarction with occlusion of the right coronary artery occurred in one patient and target lesion revascularization occurred in five patients, including two patients with severe stenosis at the ostium of the LCX only. In the remaining three patients with target lesion revascularization, two patients were treated with bare metal stents for acute myocardial infarction and, in one patient, we failed to cover the ostium of the LAD with the stent. Repeated coronary intervention to the site of restenosis at the distal stented lesion was performed in all five patients. Noncardiac death was observed in two patients. Consequently, the target lesion failure rate was $16.7 \%$, while there were no cases of in-stent restenosis of the main branch in which a drug-eluting stent covering the ostium of the LAD was successfully deployed. Kaplan-Meier curves for freedom from target lesion failure in all patients and the patients treated with drug-eluting stents are shown in Figure 2.

\section{DISCUSSION}

The present observational study evaluated the short- and long-term efficacy of the ostial stenting technique with a deep caudal projection angle. The main findings were as follows: 1) the protrusion of the stent over the ostium was very small; and 2) target lesion failure defined as

Table 2. Procedural characteristics.

\begin{tabular}{cc}
\hline Direct stent & $3(10)$ \\
Post dilation & $6(20)$ \\
RAO-caudal $40^{\circ}$ & $26(87)$ \\
Drug-eluting stent & $28(93)$ \\
Stent size (mm) & $3.43 \pm 0.22$ \\
Stent length (mm) & $25.6 \pm 6.6$ \\
Ballooning / stenting to ostial LCX & $1 / 0$ \\
\hline
\end{tabular}

Data are expressed as mean \pm SD or number (percentage). RAO denotes right anterior oblique; LCX, left circumflex artery.

Table 3. Intravascular ultrasound findings.

\begin{tabular}{cc}
\hline Baseline findings & \\
LMCA & \\
Lumen area $\left(\mathrm{mm}^{2}\right)$ & $13.57 \pm 3.59$ \\
Vessel area $\left(\mathrm{mm}^{2}\right)$ & $15.25 \pm 3.45$ \\
LAD ostial lesion & \\
Lumen area $\left(\mathrm{mm}^{2}\right)$ & $3.53 \pm 0.82$ \\
LAD reference segment & $14.22 \pm 4.64$ \\
Vessel area $\left(\mathrm{mm}^{2}\right)$ & \\
Post-procedural findings & $0.66 \pm 0.85$ \\
Mean distance $\left(\mathrm{mm}^{2}\right)$ & $20(67)$ \\
0 $<$ Distance $\leq 1.0 \mathrm{~mm}$ & $1(3)$ \\
Uncovered LAD ostium & $7.08 \pm 1.12$ \\
\hline Lumen area in LAD ostium $\left(\mathrm{mm}^{2}\right)$ &
\end{tabular}

Data are expressed as mean \pm SD or number (percentage). LMCA denotes left main coronary artery; LAD, left anterior descending artery. 
Table 4. Major adverse cardiac events during follow-up period.

\begin{tabular}{cccc}
\hline Events & $\begin{array}{c}0-180 \\
\text { days }\end{array}$ & $\begin{array}{c}181-365 \\
\text { days }\end{array}$ & $\begin{array}{c}>366 \\
\text { days }\end{array}$ \\
\hline Cardiac death & 0 & 0 & 0 \\
Non-cardiac death & 0 & 0 & 2 \\
Non-fatal myocardial infarction & 1 & 0 & 0 \\
Target lesion revascularization & 0 & 4 & 1 \\
Target vessel revascularization & 0 & 6 & 4 \\
Stent thrombosis & 0 & 0 & 0 \\
\hline
\end{tabular}

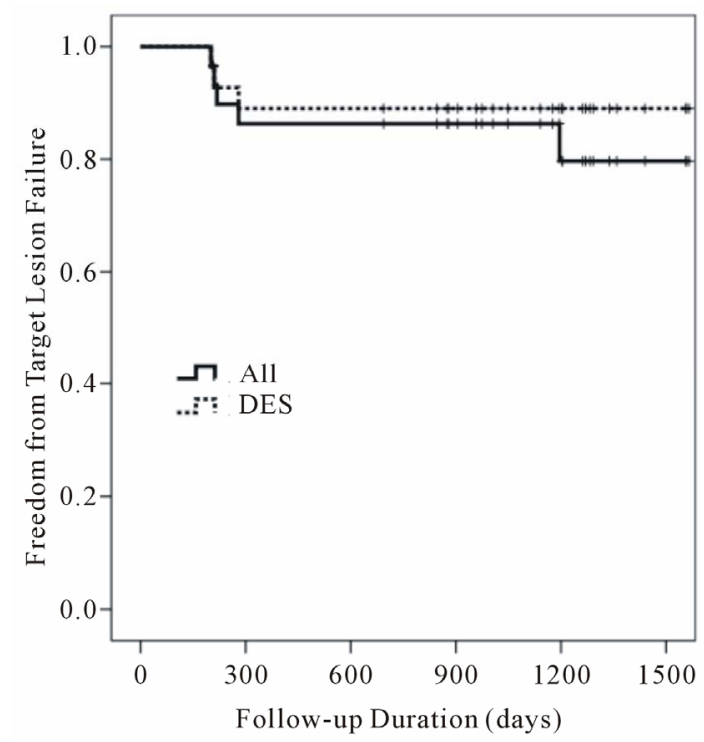

Figure 2. Kaplan-Meier curves for freedom from target lesion failure in all patients (All) and the patients treated with drug-eluting stents (DES).

cardiac death, target vessel-related nonfatal myocardial infarction, target lesion revascularization or stent thrombosis occurred in $16.7 \%$ of the patients.

The precise positioning of the stent in the ostium of the LAD is the most important technical factor in ostial LAD stenting. Several techniques have been proposed to assist in achieving accurate stent positioning in patients with ostial lesions. First, the Szabo technique anchors the stent in the ostial lesion using two wires [12]. The second wire is positioned in the aorta or the opposing branch with the most proximal end passed through the last cell of the stent inhibiting advancement, thus anchoring the stent in the accurate position. The reported success rates achieved with this technique are high [13], however, this method is technically demanding [3]. In addition, recent bench testing showed significant and asymmetric deformations in the proximal end of the stent protruding into the main branch, and the clinical data demonstrated that the technique failed to implant stents in the ostium precisely [14]. Second, the floating stent technique is a method used to deploy the proximal edge of the stent to the angiographic carina between the LAD and the LCX to guarantee complete LAD scaffolding with minimal protrusion of the stent at the LCX ostium. Medina et al. demonstrated using this technique that the mean protrusion of the stent over the origin of the LCX was $2.48 \pm$ $0.91 \mathrm{~mm}$ and that the major adverse cardiac event rate was $4 \%$ [15]. Third, IVUS-guided positioning of the stent is a simple technique that involves marking the accurate point of the ostium on a coronary angiogram [16]. The floating stent and IVUS-guided positioning techniques are essentially the same as our technique; however, protrusion of the proximal edge of the stent into the left main trunk in the present study was shorter than that observed with the floating stent technique, which suggests that clear visualization of the ostial lesions with a deep caudal projection contributes considerably to achieving an accurate stent position, even with these techniques.

We recently demonstrated the usefulness and feasibility of a 3-dimensional reconstruction system for selecting the optimal view to provide clear images of the ostial LAD. Deep caudal projection angles are optimal in most cases [11]. Tu et al. assessed obtainable optimal bifurcation viewing angles in four main coronary bifurcations and reported similar findings to ours in that the optimal view for left main bifurcation distributes primarily at the deep caudal view $\left(35^{\circ} \pm 16^{\circ}\right.$ caudal $)$ and spreads across the left anterior/right anterior oblique view $\left(4^{\circ} \pm 39^{\circ}\right.$ left anterior oblique) [17]. However, this 3-dimensional reconstruction system is not available in all catheterization laboratories, and we investigated whether the deep caudal projection is applicable for the treatment of ostial LAD lesions in daily practice without using the 3-dimensional reconstruction system. The excellent short- and long-term results obtained in the present study confirm the helpfulness of the deep caudal projection in deploying stents in the ostium of the LAD, even without using the reconstruction system.

The optimal projection angles for treating ostial lesions depend on the anatomy of the heart of each patient. Skilled operators are able to find the best view for ostial stenting without consuming radiation exposure or contrast volume. The three-dimensional reconstruction coronary angiography system also easily indicates the optimal view in the practical range. However, for most inexperienced or learning operators in catheterization laboratories unequipped with a reconstruction system, a longer procedural time and more radiation exposure and contrast are required to determine the optimal angle, and, in some cases, suboptimal projection can lead to unsuccessful results. Therefore, the use of a fixed deep caudal projection angle, which is a very simple method for all coronary interventionalists, may be not best, although it is a better strategy for implanting coronary stents in the ostium of the LAD. 
There are some important limitations associated with this study. First, this study was a single-center registry including a small number of subjects. Second, this study lacked a control group, and thus we cannot compare the results obtained with those of conventional projection angles. Moreover, we must compare our technique with the cross-over technique. Third, angiographic and clinical follow-up data were not available for some of the patients, which may have led to some bias in the present results. Finally, we used IVUS in all cases to verify the appropriateness of the deep caudal projection angles; thus, the use of IVUS may have influenced the excellent outcomes achieved with this technique.

\section{CONCLUSION}

Performing focal stent placement using a deep caudal projection is thus considered to be a feasible and effective therapeutic strategy for treating ostial lesions in the LAD.

\section{ACKNOWLEDGEMENTS}

The authors appreciate the support and collaboration of our catheterization laboratory staff. The authors have no conflicts of interest that are directly relevant to the content of this study.

\section{REFERENCES}

[1] Lemos, P.A., Hoye, A., Goedhart, D., Arampatzis, C.A., Saia, F., van der Giessen, W.J., McFadden, E., Sianos, G., Smits, P.C., Hofma, S.H., De Feyter, P.J., Van Domburg, R.T. and Serruys, P.W. (2004) Clinical, angiographic, and procedural predictors of angiographic restenosis after sirolimus-eluting stent implantation in complex patients: An evaluation from the Rapamycin-Eluting Stent Evaluated At Rotterdam Cardiology Hospital (RESEARCH) study. Circulation, 109, 1366-1370. http://dx.doi.org/10.1161/01.CIR.0000121358.26097.06

[2] Lemos, P.A., Saia, F., Ligthart, J.M., Arampatzis, C.A., Sianos, G., Tanabe, K., Hoye, A., Degertekin, M., Daemen, J., McFadden, E., Hofma, S., Smits, P.C., De Feyter, P., Van der Giessen, W.J., Van Domburg, R.T. and Serruys, P.W. (2003) Coronary restenosis after sirolimus-eluting stent implantation: Morphological description and mechanistic analysis from a consecutive series of cases. Circulation, 108, 257-260. http://dx.doi.org/10.1161/01.CIR.0000083366.33686.11

[3] Gutierrez-Chico, J.L., Villanueva-Benito, I., VillanuevaMontoto, L., Vazquez-Fernandez, S., Kleinecke, C., Gielen, S. and Iniguez-Romo, A. (2010) Szabo technique versus conventional angiographic placement in bifurcations 010001 of Medina and in aorto-ostial stenting: Angiographic and procedural results. EuroIntervention, 5, 801-808. http://dx.doi.org/10.4244/EIJV5I7A134

[4] Park, S.J., Lee, C.W., Hong, M.K., Kim, J.J. and Park, S.W. (2000) Stent placement for ostial left anterior descending coronary artery stenosis: Acute and long-term (2-year) results. Catheterization and Cardiovascular Interventions, 49, 267-271.

http://dx.doi.org/10.1002/(SICI)1522-726X(200003)49:3 $\leq 267:$ :AID-CCD9>3.0.CO;2-H

[5] Kang, S.J., Mintz, G.S., Kim, W.J., Lee, J.Y., Oh, J.H., Park, D.W., Lee, S.W., Kim, Y.H., Lee, C.W., Park, S.W. and Park, S.J. (2011) Changes in left main bifurcation geometry after a single-stent crossover technique: An intravascular ultrasound study using direct imaging of both the left anterior descending and the left circumflex coronary arteries before and after intervention. Circulation: Cardiovascular Interventions, 4, 355-361. http://dx.doi.org/10.1161/CIRCINTERVENTIONS.110.9 $\underline{61045}$

[6] Xu, J., Hahn, J.Y., Song, Y.B., Choi, S.H., Choi, J.H., Lu, C., Lee, S.H., Hong, K.P., Park, J.E. and Gwon, H.C. (2012) Carina shift versus plaque shift for aggravation of side branch ostial stenosis in bifurcation lesions: Volumetric intravascular ultrasound analysis of both branches. Circulation: Cardiovascular Interventions, 5, 657-662. http://dx.doi.org/10.1161/CIRCINTERVENTIONS.112.9 $\underline{69089}$

[7] Seung, K.B., Kim, Y.H., Park, D.W., Lee, B.K., Lee, C. W., Hong, M.K. Kim, P.J., Chung, W.S., Tahk, S.J., Park, S.W. and Park, S.J. (2005) Effectiveness of sirolimuseluting stent implantation for the treatment of ostial left anterior descending artery stenosis with intravascular ultrasound guidance. Journal of the American College of Cardiology, 46, 787-792.

http://dx.doi.org/10.1016/j.jacc.2005.06.009

[8] Cubeddu, R.J., Wood, F.O., Saylors, E.K. and Mann, T. (2007) Isolated disease of the ostium left anterior descending or circumflex artery: Management using a left main stenting technique. Clinical outcome at 2 years. Journal of Invasive Cardiology, 19, 457-461.

[9] Capranzano, P., Sanfilippo, A., Tagliareni, F., Capodanno, D., Monaco, S., Sardella, G., Giordano, A., Sangiorgi, G. M. and Tamburino, C. (2010) Long-term outcomes after drug-eluting stent for the treatment of ostial left anterior descending coronary artery lesions. American Heart Journal, 160, 973-978. http://dx.doi.org/10.1016/j.ahj.2010.07.002

[10] Kishi, K., Kimura, T., Morimoto, T., Namura, M., Muramatsu, T., Nishikawa, H., Hiasa, Y., Isshiki, T., Nobuyoshi, M. and Mitsudo, K. (2011) Sirolimus-eluting stent implantation for ostial left anterior descending coronary artery lesions: Three-year outcome from the j-Cypher Registry. Circulation: Cardiovascular Interventions, 4, 362-370.

http://dx.doi.org/10.1161/CIRCINTERVENTIONS.111.9 $\underline{61904}$

[11] Sadamatsu, K., Sagara, S., Yamawaki, T. and Tashiro, H. (2009) Three-dimensional coronary imaging for the ostium of the left anterior descending artery. The International Journal of Cardiovascular Imaging, 25, 223-228. http://dx.doi.org/10.1007/s10554-008-9385-2

[12] Kern, M.J., Ouellette, D. and Frianeza, T. (2006) A new technique to anchor stents for exact placement in ostial stenoses: The stent tail wire or Szabo technique. Catheterization and Cardiovascular Interventions, 68, 901- 
906. http://dx.doi.org/10.1002/ccd.20613

[13] Wong, P. (2008) Two years experience of a simple technique of precise ostial coronary stenting. Catheterization and Cardiovascular Interventions, 72, 331-334. http://dx.doi.org/10.1002/ccd.21558

[14] Vaquerizo, B., Serra, A., Ormiston, J., Miranda-Guardiola, F., Webber, B., Fantuzzi, A., Delgado, G. and Brugera, J. (2012) Bench top evaluation and clinical experience with the Szabo technique: New questions for a complex lesion. Catheterization and Cardiovascular Interventions, 79, 378-389. http://dx.doi.org/10.1002/ccd.23087

[15] Medina, A., Martin, P., Suarez de Lezo, J., Amador, C., Suarez de Lezo, J., Pan, M., Melian, F., Hernandez, E., Burgos, L., Ojeda, S., Ortega, J.R. and Garcia, A. (2009) Vulnerable carina anatomy and ostial lesions in the left anterior descending coronary artery after floating-stent treatment. Revista Española de Cardiología, 62, 12401249. http://dx.doi.org/10.1016/S0300-8932(09)73076-3

[16] Barbash, I.M., Waksman, R. and Kent, K.M. (2012) Utilization of intravascular ultrasound to accurately position stents in true aorto-ostial lesions. Cardiovascular Revascularization Medicine, 13, 353-356. http://dx.doi.org/10.1016/j.carrev.2012.03.001

[17] Tu, S., Jing, J., Holm, N.R., Onsea, K., Zhang, T., Adriaenssens, T., Dubois, C., Desmet, W., Thuesen, L., Chen, Y. and Reiber, J.H. (2012) In vivo assessment of bifurcation optimal viewing angles and bifurcation angles by three-dimensional (3D) quantitative coronary angiography. The International Journal of Cardiovascular Imaging, 28, 1617-1625.

http://dx.doi.org/10.1007/s10554-011-9996-x 